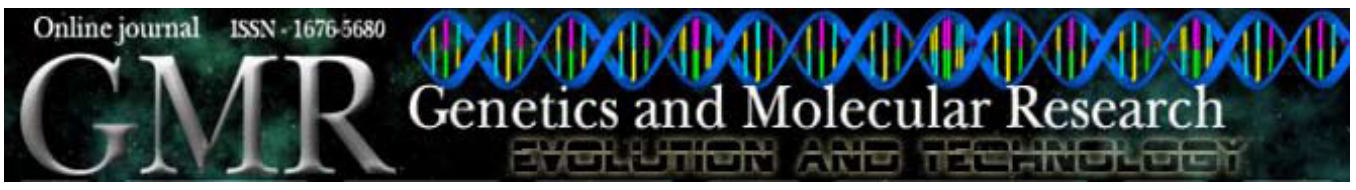

\title{
Genetic anomalies in patients with severe oligozoospermia and azoospermia in eastern Turkey: a prospective study
}

\author{
G.G. Ceylan ${ }^{1}$, C. Ceylan ${ }^{2}$ and H. Elyas ${ }^{3}$ \\ ${ }^{1}$ Ankara Atatürk Education and Training Hospital, \\ Department of Medical Genetics, Turkey \\ ${ }^{2}$ Ankara Polatlı Duatepe Public Hospital, Polatl1-Ankara, Turkey \\ ${ }^{3}$ Firat University Medical Center, Department of Medical Biology and \\ Genetics, Elazı̆̆g, Turkey \\ Corresponding author: G.G. Ceylan \\ E-mail: nil_cey@yahoo.com
}

Genet. Mol. Res. 8 (3): 915-922 (2009)

Received March 19, 2009

Accepted May 3, 2009

Published August 4, 2009

ABSTRACT. Infertility is defined as the inability to conceive a child after one year of regular unprotected intercourse; it is a major health problem affecting about $10-15 \%$ of all couples. Infertility is due to a male factor in approximately $50 \%$ of cases. The human Y chromosome contains genes necessary for gonadal differentiation into a testis and genes for complete spermatogenesis. We examined the frequency and type of both chromosomal abnormalities and Y chromosome microdeletions in 90 patients with severe male factor infertility and 75 fertile control men. Thirty of the infertile patients had nonobstructive azoospermia, 30 had oligozoospermia and 30 had normozoospermia. Five of 30 were azoospermic, four of 30 were oligozoospermic and two of 30 were normozoospermic with Y chromosome microdeletions. The AZFc locus was the most frequently deleted region (64\%). Ten cases with azoospermia, four cases with oligozoospermia and four cases with normozoospermia had chromosomal abnormalities. The 75 men with proven fertility were genetically normal. We conclude that various chromosomal 
abnormalities and deletions of the $\mathrm{Y}$ chromosome can cause infertility; therefore, genetic screening is indicated for infertile patients.

Key words: Male infertility; Y chromosome microdeletion; Chromosomal abnormality; Azoospermia; Severe oligozoospermia

\section{INTRODUCTION}

Infertility is usually described as the inability of a couple to conceive after one year of unprotected intercourse (Griffin and Finch, 2005). Infertility is a major health problem today affecting about $10-20 \%$ of couples (Mittal et al., 2004). Male factor is assumed to be responsible in about $50 \%$ of the infertile couples. Not only defects in hormone production, testicular structure, ejaculation and/or the spermatozoa themselves can adversely affect the chances of conception, but also genetic defects can affect the fertility (Griffin and Finch, 2005). The incidence of chromosome abnormalities is about ten times higher in infertile men than in the general population. Most infertile men have normal karyotypes, but their semenograms are abnormal. In many cases these patients show an increased incidence of aneuploid sperm and diploid sperm (Egozcue et al., 2003). Human Y chromosome contains genes necessary for gonadal differentiation into a testis as well as at least one gene required for full spermatogenesis (Lipshultz and Howards, 1997). The short arm of the Y chromosome (Yp) and the proximal part of the long arm of the Y chromosome (Yq) consist of euchromatin, while the distal part of the long arm is made of heterochromatin and this region may vary in length to constitute about one-half to two-thirds of Yq (Foresta et al., 2001). Y chromosome microdeletions are associated with various spermatogenic alterations, including Sertoli cell-only (SCO) syndrome, maturation arrest and hypospermatogenesis (Van Golde et al., 2001). The microdeletions of the long arm of Y chromosome can be the pathogenic reason of male infertility (Mittal et al., 2004). The Y chromosome has been divided into seven deletion intervals. Each of these intervals is further subdivided into subintervals. In 1992, a 43-interval deletion map of a human Y chromosome that contained an ordered array of sequence tagged sites (STS) had been shown, which spanned the entire length of the $\mathrm{Y}$ chromosome. The genes critical for spermatogenesis are located on the long arm of the $Y$ chromosome in deletion interval 5 and 6 band 11.23. This region is referred to as the AZF, as the most severe phenotype associated with its deletion is azoospermia. The AZF region has three nonoverlapping loci - AZFa, AZFb, and AZFc - deletions of which are associated with spermatogenic failure. The AZFa locus is located on proximal Yq11 (Yq11.21), while AZFb and AZFc are located on distal Yq11 (Yq11.23). Deletion of these loci results in spermatogenic arrest and is associated with azoospermia, oligozoospermia, and also with a varied testis histological profile ranging from SCO, to hypospermatogenesis, to maturation arrest (Dada et al., 2004). Some of the genes in the Yq11 region control the spermatogenesis. These genes are localized in the Yq11 region, in the 5-6. deletion interval and in the 11.23 band (Van Golde et al., 2001).

Microdeletions of the $\mathrm{Y}$ chromosome removing the azoospermia factor (AZF) region or parts thereof are found in men suffering from azoospermia or oligozoospermia and are the second most frequent genetic cause of spermatogenic failure after Klinefelter's syndrome (Hucklenbroich et al., 2005). A fourth AZF region between AZFb and AZFc, termed as AZFd, has been found. Patients with microdeletions restricted to AZFd may present mild oligozoo- 
spermia or even normal sperm counts associated with abnormal sperm morphology. Though a definitive genotype/phenotype correlation does not exist, large deletions spanning multiple AZF regions or microdeletions restricted to AZFa usually result in patients with SCO or severe oligozoospermia, whereas microdeletions restricted to $\mathrm{AZFb}$ or $\mathrm{AZFc}$ can result in patients with phenotypes, which range from SCO to moderate oligozoospermia (De Palma et al., 2005).

Chromosome abnormalities can also cause male infertility. Chromosome abnormalities can be broken down into structural and numerical types. Structural abnormalities include inversions and balanced translocations. Inversions can be paracentric or pericentric. Robertsonian or reciprocal translocations may cause male infertility. Reciprocal translocations are found more in oligozoospermic patients $(1.7 \%)$ than in azoospermic patients $(0.6 \%)$. Robertsonian translocations are also found more in oligozoospermic patients $(0.9 \%)$ than azoospermic patients $(0.3 \%)$. Numerical abnormalities include constitutional trisomy (mosaic or non-mosaic). A second type of numerical abnormality is aneuploidy confined to the sperm (De Palma et al., 2005; Lissitsina et al., 2006).

Various chromosomal abnormalities and deletions of Y chromosome can cause infertility, therefore genetic screening must be suggested to infertile patients. In this prospective study, we aimed to show to clinicians the importance of genetic screening in male infertility.

\section{MATERIAL AND METHODS}

Infertile men $(\mathrm{N}=90)$ were prospectively recruited for chromosomal analysis from 2004 to 2007 at Firat University Medical Center, Department of Medical Biology and Genetics. The patients applied because of severe male-factor infertility and past history of infertility for more than two years were selected. The mean age of the patients was 30.8 years (range 20-44 years). All underwent an andrological work-up, which included medical history, physical examination, hormonal estimation (excluding those with obstructive azoospermia) and semen analysis according to World Health Organization recommendations and standards (Tuarnaye, 2002). There were 30 men with non-obstructive azoospermia, 30 men with severe oligozoospermia with a sperm count of $<5 \times 10^{6} / \mathrm{mL}$ and 30 men with normozoospermia and primary infertility. Seventy-five normozoospermic male donors with normal semen parameters (sperm count $>20 \times 10^{6} / \mathrm{mL}$, progressive motility $>50 \%$ and normal morphology $>30 \%$ ) and proven fertility were included as controls. None of them had any history of childhood disease, environmental exposure, radiation exposure, or prescription drug usage that could account for their infertility. Informed consent was taken from the patients and donors prior to collection of heparinised blood samples. Chromosome investigations were performed on cultures of peripheral blood lymphocytes using standard techniques (Barch et al., 1997). From each patient, 50 wellspread metaphases were analyzed by G-banding. All chromosomal abnormalities have been reported in accordance with the current international standard nomenclature (Shaffer and Tommerup, 2005). To screen for microdeletion in the AZF region of the Y chromosome by polymerase chain reaction (PCR), genomic DNA was prepared from the peripheral blood samples. Each man was analyzed for the presence of STS in the AZFa, AZFb, and AZFc regions. The STS probes used were sY84 and sY86 (AZFa), sY127, sY133 and sY134 (AZFb), sY254 and sY255 (AZFc), and SRY and ZFX/ZFY (controls). In addition, a water sample that contains all reaction components but water instead of DNA was used 
for reagent contamination. PCR conditions used for STS markers were as follows: initial denaturation at $94^{\circ} \mathrm{C}$ for $5 \mathrm{~min}$ and subsequent denaturations at $94^{\circ} \mathrm{C}$ for $30 \mathrm{~s}$ were the same for all samples. Annealing was $56^{\circ} \mathrm{C}$ for $40 \mathrm{~s}$, extension was $65^{\circ} \mathrm{C}$ for $4 \mathrm{~min}$. Subsequent series were 35 cycles. Final extension was carried out at $72^{\circ} \mathrm{C}$ for $5 \mathrm{~min}$. The PCR products were separated on a $2 \%$ agarose gel stained with ethidium bromide and visualized using ultraviolet illumination. A PCR of the expected size was scored positive for the given STS, and scored negative if no product was detectable after 3 successive PCR.

\section{RESULTS}

SCO syndrome was determined in 12 azoospermic patients (12\%), maturation arrest in 10 azoospermic patients (33.3\%) and generalized fibrosis in 8 azoospermic patients (26.7\%) in testis biopsies. Among 30 azoospermic infertile men studied, 10 performed some kind of constitutional chromosomal abnormality corresponding to a frequency of $33.3 \%$. Klinefelter syndrome was found in $8(26.7 \%)$ azoospermic patients, 47 ; XYY syndrome was found in 1 azoospermic patient $(3.3 \%)$ and $46, \mathrm{XY}, \mathrm{Yqh}(+)$ in 1 azoospermic patient $(3.3 \%)$. The occurrence of chromosomal abnormality in the azoospermics, oligozoospermics and normozoospermics was $33.3,13.3$ and $10 \%$, respectively. A normal karyotype of 46,XY was observed in all controls. The karyotypes of the patients according to the groups are shown in Table 1.

\begin{tabular}{|c|c|c|c|c|}
\hline Karyotypes & Azoospermics & Oligozoospermics & Normospermic infertiles & Control \\
\hline 46,XY(normal) & $20(66.7 \%)$ & $26(86.7 \%)$ & $27(90 \%)$ & $75(100 \%)$ \\
\hline $47, \mathrm{XXY}$ & $8(26.7 \%)$ & $1(3.3 \%)$ & & \\
\hline 46,XY,Yqh(+) & $1(3.3 \%)$ & $2(6.7 \%)$ & $2(6.7 \%)$ & \\
\hline $47, \mathrm{XYY}$ & $1(3.3 \%)$ & - & - & \\
\hline $46, X Y, \operatorname{inv}(9)$ & - & $1(3.3 \%)$ & - & \\
\hline $\operatorname{inv}(9)$ & - & - & $1(3.3 \%)$ & \\
\hline $46, X Y, \operatorname{inv}(9)$ & - & - & $-\quad-$ & \\
\hline Total & $30(100 \%)$ & $30(100 \%)$ & $30(100 \%)$ & \\
\hline
\end{tabular}

Data are reported as frequency with percent in parentheses.

$\mathrm{Y}$ chromosome microdeletion was determined in 5 patients in the azoospermic group, 4 patients in the severe oligozoospermic group and 2 patients in the normozoospermic group. Microdeletions in the AZFc region (63.6\%) were encountered more often than in AZFb $(54.5 \%)$ and in AZFa (9.1\%) in the azoospermic group. Deletion of AZFb+c (50\%), AZFb $(25 \%)$ and AZFa (25\%) were determined in oligozoospermic group. Figure 1 shows a patient with deletion of AZFc region. Hormone profiles were high in the azoospermic group and normal in the oligozoospermic group. The clinical features, hormone profiles and Yq microdeletions of the patients are shown in Table 2. The frequencies of patient groups are shown in Table 3. Yates-chi-square test was used for the comparison of patient and control groups. The differences for chromosomal abnormalities and $\mathrm{Y}$ microdeletions between the control group and the patient group were statistically significant $(\mathrm{P}<0.05)$. 
Table 2. The clinical features, hormone profiles and Yq microdeletions of the patients.

\begin{tabular}{lccccc}
\hline Patient & Sperm count & Hormone profile & Histopathologic findings & Karyotype & Deleted region \\
\hline A5 & Azoospermic & High & SCOS & $47, X X Y$ & AZFc \\
A6 & Azoospermic & High & MA & $46, X Y, Y q h(+)$ & AZFb \\
A10 & Azoospermic & High & SCOS & $46, X Y$ & AZFc \\
A12 & Azoospermic & High & SCOS & $46, X Y$ & AZFa, AZFc \\
A15 & Azoospermic & High & MA & $46, X Y$ & AZFb, AZFc \\
O3 & Oligozoospermic & Normal & - & $46, X Y$ & AZFc \\
O16 & Oligozoospermic & Normal & - & $46, X Y$ & AZFb, AZFc \\
O18 & Oligozoospermic & Normal & - & $46, X Y$ & AZFb \\
O26 & Oligozoospermic & Normal & - & $46, X Y$ & AZFb, AZFc \\
N5 & Normospermic & Normal & - & $46, X Y, Y q h(+)$ & AZFb \\
N15 & Normospermic & Normal & - & $46, X Y$ & AZFb \\
\hline
\end{tabular}

$\mathrm{SCOS}=$ Sertoli cell-only syndrome; MA = maturation arrest.

Table 3. The frequencies of $\mathrm{Y}$ microdeletions and chromosomal abnormalities in patient groups.

\begin{tabular}{lccrrr}
\hline & & Azoospermia & Oligozoospermia & Normospermics & Total \\
\hline Y chromosome microdeletion & + & $5(16.7 \%)$ & $4(13.3 \%)$ & $2(6.7 \%)$ & $11(12.2 \%)$ \\
& - & $25(83.3 \%)$ & $26(86.7 \%)$ & $28(93.3 \%)$ & $79(87.8 \%)$ \\
Chromosomal abnormality & + & $10(33.3 \%)$ & $4(13.3 \%)$ & $3(10 \%)$ & $17(18.9 \%)$ \\
& - & $20(66.7 \%)$ & $26(86.7 \%)$ & $27(90 \%)$ & $73(81.1 \%)$ \\
Genetic anomaly & + & $15(50 \%)$ & $8(26.7 \%)$ & $5(16.7 \%)$ & $28(31.1 \%)$ \\
& - & $15(50 \%)$ & $22(73.3 \%)$ & $25(83.3 \%)$ & $62(68.9 \%)$ \\
\hline
\end{tabular}

Data are reported as frequency with percent in parentheses.

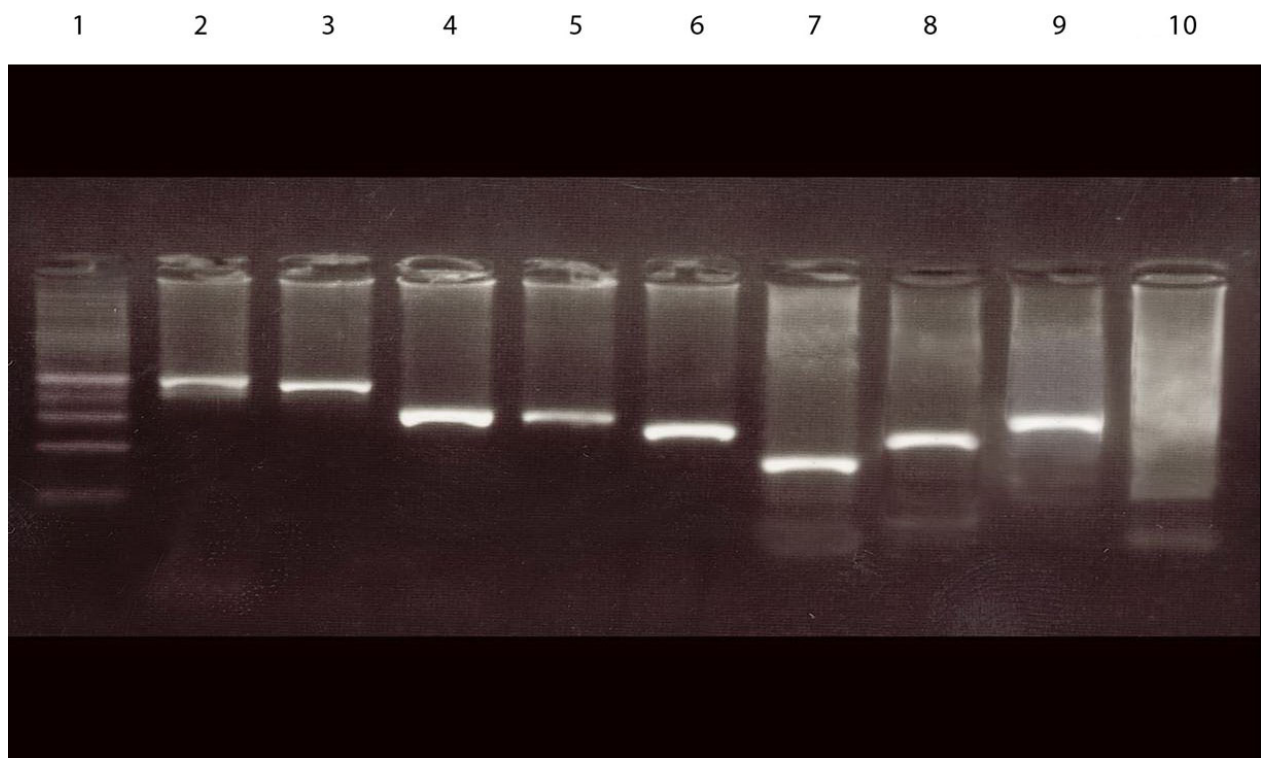

Figure 1. The image of azoospermic patient with a deletion of $A Z F c$ region (sY255: there is no band). Lane $1=$ DNA ladder for $100 \mathrm{bp}$; lane $2=\mathrm{ZFY} / \mathrm{ZFX}$; lane $3=\mathrm{SRY}$; lane $4=\mathrm{sY84}$; lane $5=\mathrm{sY86}$; lane $6=\mathrm{sY} 127$; lane 7 $=\mathrm{sY} 133$; lane $8=\mathrm{sY} 134$; lane $9=\mathrm{sY} 254$; lane $10=\mathrm{sY} 255$. 


\section{DISCUSSION}

Male factor is assumed to be responsible in $50 \%$ of the infertile couples (O'Connell et al., 2002). Recent studies have focused on the genetic basis of male infertility (Vicdan et al., 2004). Cytogenetic anomalies and $\mathrm{Y}$ chromosome microdeletions are responsible from male infertility in a rate of $60 \%$. There are chromosomal abnormalities in $13.7 \%$ of azoospermic patients and $4.6 \%$ of oligozoospermic patients; this ratio is $5.1 \%$ of total infertile male population (Quilter et al., 2003). Chromosomal abnormality is higher in azoospermic patients (13.7-15.4\%) than severe oligozoospermic patients (1.7-4.6\%). Sex chromosome abnormalities predominate in azoospermic patients $(12.6 \%)$ whereas autosomal abnormalities are the most frequent in the severe oligozoospermic patients (Vicdan et al., 2004).

In this study, chromosomal abnormality incidence has been found as $16.7 \%$ in the azoospermic group and $13.3 \%$ in the severe oligozoospermic group. As mentioned in the literature, chromosomal abnormality incidence was higher in the azoospermic group than in the oligozoospermic group; this shows that the incidence of chromosomal abnormality increases as sperm count decreases. In the azoospermic group, sex chromosome abnormalities were found only in the severe oligozoospermic group, sex and autosomal chromosome abnormalities were found together. Klinefelter syndrome was found in 8 patients, XYY syndrome in 1 patient and $46, \mathrm{XY}, \mathrm{Yqh}(+)$ in one patient of the azoospermic group. In the severe oligozoospermic group, Klinefelter syndrome was found in one patient, 46,XY,Yqh(+) in 2 patients and homozygote pericentric inversion of chromosome 9 in one patient. $46, \mathrm{XY}, \mathrm{Yqh}(+)$ in two patients and $46, X Y, \operatorname{inv}(9)$ in one patient were found in the normozoospermic group. One of the parents of the carriers of patients with pericentric inversion of chromosome 9 was also carrier, while both of the parents of the homozygote patient were carriers. The differences for chromosomal abnormalities between the control group and the patient group were statistically significant $(\mathrm{P}<0.05)$.

Among infertile men, the prevalance of $\mathrm{Y}$ microdeletions is approximately $7 \%$ (1$35 \%)$. A small portion ( $2 \%$ ) of the fertile male population might also harbor very small microdeletions of the $\mathrm{Y}$ chromosome, involving noncoding regions (Choi et al., 2004). In a study by Şamlı et al. (2006), 383 azoospermic and 436 oligozoospermic patients were analyzed and 4 of $436(0.9 \%)$ oligozoospermic patients were found to have Klinefelter syndrome. Although the heterozygote inversion of chromosome 9 is accepted as a polymorphism, homozygosity is thought to be associated with primary infertility (Şamlı et al., 2006).

Tiepolo and Zuffardi (1976) reported on the cytogenetic analysis of 1160 infertile men and found that $0.5 \%$ had macroscopic deletions of the distal long arm of the Y chromosome. Thus, they suggested the presence of an AZF in the distal euchromatic region of Yq. Karyotypic analysis was significantly improved with the advent of PCR (Tiepolo and Zuffardi, 1976; Girardi et al., 1997). Chandley and Cooke (1994) studied 50 azoospermic and oligozoospermic men and found a deletion in the Yq region for four men. Prior studies have proposed that $3-18 \%$ of men with nonobstructive azoospermia or severe oligozoospermia may have deletions of the $Y$ chromosome (Girardi et al., 1997). In a study of 340 azoospermic patients, $Y$ chromosome microdeletions were found in 29 patients $(8.5 \%)$ and most of the deletions were in the AZFc region (82.8\%), the AZFb region (55.\%) and the AZFa region (24.1\%). Microdeletions were investigated in AZFa region, and macrodeletions were found in AZFb and AZFc regions. In the same study, histological investigation of the testicular tissue of the azoospermic 
men with Y chromosome microdeletion revealed complete absence of germ cells and presence of SCO (Thangaraj et al., 2003).

Oliva et al. (1998) reviewed the published series of azoospermia and demonstrated that the worldwide frequency of microdeletions was $13 \%$. Choi et al. (2004) showed that TESE retrieval rates for the Y-microdeleted men are not significantly different from those of patients with idiopathic nonobstructive azoospermia. Most AZF deficiencies are de novo events due to deletions occurring in germ cells or in post-zygotic stages. Germ cell deletions generate a mosaic sperm population carrying normal and AZF-deleted Y chromosomes. PCR deletion analyses of these cases usually show extensive AZF deficiencies involving several loci in the same or in adjacent AZF subintervals (Kent-First et al., 1999; De Palma et al., 2005). Vogt et al. (1996) have suggested that the deletions in the AZFa and AZFb are associated with the impairment of spermatogenesis being worse when compared with that of the AZFc region. Ferlin et al. (1999) reported a high frequency of $\mathrm{Y}$ deletions in complete absence or strong reduction of germ cells, while milder forms of testiculopathy were not associated with Y deletions. Silber et al. (1998) have suggested that when AZFb and beyond were missing, there was no detectable completion of spermatogenesis. This correlation has been supported by many other studies, but some others have not supported this correlation.

Krausz and McElreavey (2001) reported that there were microdeletions outside of $\mathrm{DAZ}$ region in 12 of 229 control individuals. The complete deletions of the AZFa and AZFb regions are associated with SCO syndrome and spermatogenic arrest (Kostiner et al., 1998). Deletions of the AZFc region are usually associated with hypospermatogenesis and SCO syndrome type II (Krausz et al., 2000).

It is known that deletion frequency is different between the subgroups of sperm count (severe oligozoospermic and azoospermic) and etiology (idiopathic and non-idiopathic). Microdeletions are rarely found in a large control group of men with proven fertility; it has been shown that there is a specific correlation between deletions and spermatogenesis (Simoni et al., 2004). In most studies reporting a high frequency of Y chromosome microdeletions, a selected group of infertile men with severe spermatogenesis failure was included. On the other hand, there are only a few studies analyzing unselected men consecutively attending infertility clinics. Therefore, to establish the clinical importance of Y chromosome microdeletions in the evaluation of male infertility, studies analyzing unselected groups of infertile men are needed (Medica et al., 2005).

The studies in the future will be on other fertility genes except from the ones on Y chromosome. As the time passes more and more autosomal and sex linked genes are established. The data about molecular genetics and human genome project will be an excellent basis on the area of male fertility.

\section{REFERENCES}

Barch MJ, Knutsen T and Spurbeck JL (1997). The AGT Cytogenetics Laboratory Manual. 3rd edn. Lippincott-Raven, Philadelphia.

Chandley AC and Cooke HJ (1994). Human male fertility-Y-linked genes and spermatogenesis. Hum. Mol. Genet. 3: 1449-1452.

Choi JM, Chung P, Veeck L, Mielnik A, et al. (2004). AZF microdeletions of the Y chromosome and in vitro fertilization outcome. Fertil. Steril. 81: 337-341.

Dada R, Gupta NP and Kucheria K (2004). Yq microdeletions - azoospermia factor candidate genes and spermatogenic arrest. J. Biomol. Tech. 15: 176-183. 
De Palma A, Burrello N, Barone N, D'Agata R, et al. (2005). Patients with abnormal sperm parameters have an increased sex chromosome aneuploidy rate in peripheral leukocytes. Hum. Reprod. 20: 2153-2156.

Egozcue J, Blanco J, Anton E, Egozcue S, et al. (2003). Genetic analysis of sperm and implications of severe male infertility - a review. Placenta 24 (Suppl B): S62-S65.

Ferlin A, Moro E, Garolla A and Foresta C (1999). Human male infertility and Y chromosome deletions: role of the AZFcandidate genes DAZ, RBM and DFFRY. Hum. Reprod. 14: 1710-1716.

Foresta C, Moro E and Ferlin A (2001). Y chromosome microdeletions and alterations of spermatogenesis. Endocr. Rev. 22: $226-239$

Girardi SK, Mielnik A and Schlegel PN (1997). Submicroscopic deletions in the Y chromosome of infertile men. Hum. Reprod. 12: 1635-1641.

Griffin DK and Finch KA (2005). The genetic and cytogenetic basis of male infertility. Hum. Fertil. 8: 19-26.

Hucklenbroich K, Gromoll J, Heinrich M, Hohoff C, et al. (2005). Partial deletions in the AZFc region of the Y chromosome occur in men with impaired as well as normal spermatogenesis. Hum. Reprod. 20: 191-197.

Kent-First M, Muallem A, Shultz J, Pryor J, et al. (1999). Defining regions of the Y-chromosome responsible for male infertility and identification of a fourth AZF region (AZFd) by Y-chromosome microdeletion detection. Mol. Reprod. Dev. 53: 27-41.

Kostiner DR, Turek PJ and Reijo RA (1998). Male infertility: analysis of the markers and genes on the human Y chromosome. Hum. Reprod. 13: 3032-3038.

Krausz C and McElreavey K (2001). Y chromosome microdeletions in 'fertile' males. Hum. Reprod. 16: 1306-1307.

Krausz C, Quintana-Murci L and McElreavey K (2000). Prognostic value of Y deletion analysis: what is the clinical prognostic value of Y chromosome microdeletion analysis? Hum. Reprod. 15: 1431-1434.

Lipshultz LI and Howards SS (1997). Infertility in the Male. 3rd edn. Mosby Year Book Inc., Saint Louis.

Lissitsina J, Mikelsaar R and Punab M (2006). Cytogenetic analyses in infertile men. Arch. Androl. 52: 91-95.

Medica I, Gligorievska N, Prenc M and Peterlin B (2005). Y microdeletions in the Istria county, Croatia. Asian J. Androl. 7: 213-216.

Mittal RD, Singh G, Srivastava A, Pradhan M, et al. (2004). Y chromosome micro-deletions in idiopathic infertility from Northern India. Ann. Genet. 47: 331-337.

O'Connell M, McClure N and Lewis SE (2002). Mitochondrial DNA deletions and nuclear DNA fragmentation in testicular and epididymal human sperm. Hum. Reprod. 17: 1565-1570.

Oliva R, Margarit E, Ballesca JL, Carrio A, et al. (1998). Prevalence of Y chromosome microdeletions in oligospermic and azoospermic candidates for intracytoplasmic sperm injection. Fertil. Steril. 70: 506-510.

Quilter CR, Svennevik EC, Serhal P, Ralph D, et al. (2003). Cytogenetic and Y chromosome microdeletion screening of a random group of infertile males. Fertil. Steril. 79: 301-307.

Şamlı H, Şamlı MM, Solak M and Imirzalioglu N (2006). Genetic anomalies detected in patients with non-obstructive azoospermia and oligozoospermia. Arch. Androl. 52: 263-267.

Shaffer LG and Tommerup N (2005). An International System for Human Cytogenetic Nomenclature. 1st edn. S. Karger, Basel, 82.

Silber SJ, Alagappan R, Brown LG and Page DC (1998). Y chromosome deletions in azoospermic and severely oligozoospermic men undergoing intracytoplasmic sperm injection after testicular sperm extraction. Hum. Reprod. 13: 3332-3337.

Simoni M, Bakker E and Krausz C (2004). EAA/EMQN best practice guidelines for molecular diagnosis of Y-chromosomal microdeletions. State of the art 2004. Int. J. Androl. 27: 240-249.

Thangaraj K, Gupta NJ, Pavani K, Reddy AG, et al. (2003). Y chromosome deletions in azoospermic men in India. $J$. Androl. 24: 588-597.

Tiepolo L and Zuffardi O (1976). Localization of factors controlling spermatogenesis in the nonfluorescent portion of the human Y chromosome long arm. Hum. Genet. 34: 119-124.

Tuarnaye H (2002). Gamete Source and Manipulation. In: Current Practices and Controversies in Assisted Reproduction: Report of a WHO Meeting (Vayana E, Rowe PS and Griffin PD, eds.). WHO (World Health Organization), Geneva, 83-101.

Van Golde RJ, Wetzels AM, de Graaf R, Tuerlings JH, et al. (2001). Decreased fertilization rate and embryo quality after ICSI in oligozoospermic men with microdeletions in the azoospermia factor $\mathrm{c}$ region of the Y chromosome. Hum. Reprod. 16: 289-292.

Vicdan A, Vicdan K, Gunalp S, Kence A, et al. (2004). Genetic aspects of human male infertility: the frequency of chromosomal abnormalities and Y chromosome microdeletions in severe male factor infertility. Eur. J. Obstet. Gynecol. Reprod. Biol. 117: 49-54.

Vogt PH, Edelmann A, Kirsch S, Henegariu O, et al. (1996). Human Y chromosome azoospermia factors (AZF) mapped to different subregions in Yq11. Hum. Mol. Genet. 5: 933-943. 